\title{
ON TWO PARAMETER SINGULAR PERTURBATION OF LINEAR BOUNDARY VALUE PROBLEMS
}

\author{
W. M. GREENLEE ${ }^{1}$
}

\begin{abstract}
A two parameter perturbation estimate for solutions of a functional equation in Hilbert space is derived. The estimate is applied to two parameter singular perturbation of elliptic boundary value problems with homogeneous Dirichlet boundary data.
\end{abstract}

1. Consider the boundary value problem

$$
\epsilon A u+\mu C u+B u=f \equiv f_{e, \mu}
$$

for $0<\epsilon \leqq \epsilon_{0}$ and $0 \leqq \mu \leqq \mu_{0}$, where $A$ and $B$ are linear elliptic differential operators of respective orders $2 m^{\prime}>2 m$ over a bounded domain $D$; and $C$ is a linear differential operator of order $\leqq 2 m^{\prime}$. The solution $u$ of the above equation is to be compared with the solution $u_{0}$ of $B u_{0}=f_{0}$ where $f \rightarrow f_{0}$ as $\epsilon \downarrow 0$ and $\mu \downarrow 0$. In particular, bounds of the form $\left|u-u_{0}\right|_{m, D}=o\left(\epsilon^{\tau}\right)+o\left(\mu^{\sigma}\right)$ in the Bessel potential space $P^{m}(D)$ will be derived, assuming a like bound for the $P^{-m}(D)$ norm of $f-f_{0}$.

For the one parameter problem obtained by setting $C=0$ above, corresponding bounds have been obtained by Friedman [4], Greenlee [5] and Huet [8], [9]. Extensive studies in multiparameter singular perturbation theory have been carried out by O'Malley, cf. [12]. In Greenlee [6] a two parameter perturbation problem analogous to the above, but with $C$ a quasilinear differential operator of order $\leqq 2 m$, was considered.

In this paper a perturbation theorem for a functional equation in abstract Hilbert space is proven. The theorem is then applied to differential problems of the type described above. The notation and methods of this paper are similar to those used in [5] and [6].

2. Let $V$ and $V_{0}$ be complex Hilbert spaces with $V C_{c} V_{0}$, and $V$ dense in $V_{0}$. Denote by $|v|_{V},(v, w)_{V},|v|_{0}$ the norms and inner products in $V$ and $V_{0}$ respectively. Let $a(v, w), c(v, w)$ be continuous Hermitian bilinear (sesquilinear) forms on $V$ and let $b(v, w)$ be a continuous Hermitian bilinear form on $V_{0}$. Further assume that:

Received by the editors March 6, 1970.

AMS 1969 subject classifications. Primary 3514, 4748; Secondary 3545.

Key words and phrases. Two parameter singular perturbation, Hilbert space, quadratic interpolation, Bessel potential, elliptic boundary value problem.

${ }^{1}$ Research supported in part by NSF Grant GP-9651. 
(1) there exists $\beta>0$ such that $|b(v, v)| \geqq \beta|v|_{0}^{2}$ for all $v \in V_{0}$; and,

(2) for $0<\epsilon \leqq \epsilon_{0}$ and $0 \leqq \mu \leqq \mu_{0}$ there exist $\alpha(\epsilon, \mu)>0$ such that $\alpha(\epsilon, \mu) \rightarrow 0$ as $\epsilon \downarrow 0$ and $\delta>0$ such that

$$
|\epsilon a(v, v)+\mu c(v, v)+b(v, v)| \geqq \alpha(\epsilon, \mu)|v|_{V}^{2}+\delta|v|_{0}^{2} \text { for all } v \in V .
$$

According to a theorem of Hausdorff (cf. Aronszajn [3]) the inequality in (2) holds if and only if there is an angle $\psi \equiv \psi(\epsilon, \mu)$ such that

$$
\operatorname{Re}\left\{e^{i \psi}[\epsilon a(v, v)+\mu c(v, v)+b(v, v)]\right\} \geqq \alpha(\epsilon, \mu)|v|_{V}^{2}+\delta|v|_{0}^{2} .
$$

The dependence of the angle $\psi$ on $\epsilon$ and $\mu$ does not seem easy to analyze. Simpler conditions implying (1) and the inequality in (2) are (cf. Huet [7, Theorem 1.4]) the existence of a fixed angle $\phi$ such that

(i) $\operatorname{Re}\left(e^{i \phi} b(v, v)\right) \geqq \beta|v|_{0}^{2}$

(ii) $\operatorname{Re}\left(e^{i \phi} c(v, v)\right) \geqq 0$, and

(iii) $\operatorname{Re}\left\{e^{i \phi}[\epsilon a(v, v)+b(v, v)]\right\} \geqq k(\epsilon)|v|_{V}^{2}, k(\epsilon)>0$.

For differential problems (i) and (iii) are strong Gårding type inequalities.

Let $V_{0}^{*}$ be the antidual of $V_{0}$, and let $L_{\epsilon, \mu} \equiv L, 0<\epsilon \leqq \epsilon_{0}, 0 \leqq \mu \leqq \mu_{0}$, and $L_{0}$ be given in $V_{0}^{*}$. It follows from the Lax-Milgram lemma that the equation

$$
b\left(u_{0}, v\right)=L_{0}(v) \quad \text { for all } v \in V_{0}
$$

has a unique solution $u_{0} \in V_{0}$. Similarly denote by $x$ the unique solution in $V$ of

$$
\epsilon a(x, v)+\mu c(x, v)+b(x, v)=L_{0}(v) \quad \text { for all } v \in V,
$$

and by $u$ the unique solution in $V$ of

$$
\epsilon a(u, v)+\mu c(u, v)+b(u, v)=L(v) \quad \text { for all } v \in V .
$$

In each of (4), (5) it is assumed that $0<\epsilon \leqq \epsilon_{0}$ and $0 \leqq \mu \leqq \mu_{0}$.

As in [5, p. 142], let $Q$ be the operator in $V_{0}$ associated with $a(v, w)$ relative to $b(v, w)$, i.e., $a$ is defined by $b(a v, w)=a(v, w)$ for all $w \in V$ on $D(Q)=\{v \in V: w \rightarrow a(v, w)$ is continuous on $V$ in the topology of $\left.V_{0}\right\}$. $a$ is a closed densely defined operator in $V_{0}$ (cf. [5, Proposition 2.1]). Moreover, $D(a)$, provided with the graph norm $\left(|v|_{0}^{2}+|a v|_{0}^{2}\right)^{1 / 2}$, is a Hilbert space, $V_{1}$, which is dense in $V$ and whose norm and inner product will be denoted by $|v|_{1},(v, w)_{1}$, respectively. 
The interpolation spaces by quadratic interpolation between $V_{1}$ and $V_{0}$ will be denoted by $V_{r}, 0 \leqq \tau \leqq 1$, (cf. Lions [10]) with norm $|v|_{\tau}$.

Now let $C$ be the operator in $V_{0}$ associated with $c(v, w)$ relative to $b(v, w)$ and assume that

(6) $D(C) \supset D(\propto)$, and

(7) there is a $\gamma \in[0,1]$ and $k>0$ such that

$$
|C v|_{0} \leqq k|v|_{\gamma} \text { for all } v \in V_{1}=D(a) \text {. }
$$

It follows from (6) that for $\epsilon \in\left(0, \epsilon_{0}\right]$ and $\mu \in\left[0, \mu_{0}\right], \epsilon Q+\mu C+I$ is the operator in $V_{0}$ associated with $\epsilon a(v, w)+\mu c(v, w)+b(v, w)$ relative to $b(v, w)$. Furthermore, $\epsilon \mathfrak{Q}+\mu C+I$ is a closed operator in $V_{0}$ which is a topological isomorphism of its domain, $D(a)$, onto $V_{0}$.

For a real valued function $g(\epsilon, \mu)$ the notation $g(\epsilon, \mu)=o(\epsilon)+O(\mu)$ will signify that $|g|$ is dominated by the sum of a function of $\epsilon$ which is $o(\epsilon)$ as $\epsilon \downarrow 0$ and a function of $\mu$ which is $O(\mu)$ as $\mu \downarrow 0$. Then the following rate of convergence theorem describes the behavior of $u$ (and $x$ ) as $\epsilon \downarrow 0$ and $\mu \downarrow 0$.

Theorem. Assume hypotheses (1), (2), (6), (7) and let $u_{0}, u$ be the solutions of (3), (5) respectively. Then one has:

(i) if $u_{0} \in V_{1}=D(Q)$ and $\left\|L-L_{0}\right\|=O(\epsilon)+O(\mu)$, then $\left|u-u_{0}\right|_{0}$ $=O(\epsilon)+O(\mu)$;

(ii) if $\gamma \in[0,1)$ and for fixed $\tau \in[\gamma, 1), u_{0} \in V_{\tau}$ and $\left\|L-L_{0}\right\|=o\left(\epsilon^{\tau}\right)$ $+O(\mu)$, then $\left|u-u_{0}\right|_{0}=o\left(\epsilon^{\tau}\right)+O(\mu)$;

(iii) if for fixed $\tau \in[0, \gamma), u_{0} \in V_{\tau}$ and $\left\|L-L_{0}\right\|=o\left(\epsilon^{\tau}\right)+o\left(\mu^{\tau / \gamma}\right)$, then $\left|u-u_{0}\right|_{0}=o\left(\epsilon^{\tau}\right)+o\left(\mu^{\tau / \gamma}\right)$.

Proof. Subtraction of (4) from (5) yields

$$
\epsilon a(u-x, v)+\mu c(u-x, v)+b(u-x, v)=\left(L-L_{0}\right)(v)
$$

for all $v \in V$. By letting $v=u-x$ it follows from (2) that

$$
\alpha(\epsilon, \mu)|u-x|_{V}^{2}+\delta|u-x|_{0}^{2} \leqq\left\|L-L_{0}\right\| \cdot|u-x|_{0} .
$$

Hence $|u-x|_{0} \leqq(1 / \delta)\left\|L-L_{0}\right\|$, and so

$$
\left|u-u_{0}\right|_{0} \leqq(1 / \delta)|| L-L_{0} \|+\left|x-u_{0}\right|_{0} .
$$

It is thus sufficient to prove that (i)-(iii) hold with $u$ replaced by $x$.

For this purpose observe that by (4), (6) and the definitions of $a$ and $C, x$ is the unique solution in $V_{1}=D(Q)$ of

$$
(\epsilon \mathfrak{Q}+\mu C+I) x=u_{0} .
$$

Now with $\Lambda=a^{*} a+I$ and $S=\Lambda^{1 / 2},\left(\epsilon S+\mu S^{\gamma}+I\right)^{-1}$ is a bounded 
linear operator on $V_{0}$. Let $y$ be the unique solution in $D(S)=D(a)$ of

$$
\left(6 S+\mu S^{\gamma}+1\right) y=u_{0} \text {. }
$$

An estimate of the form

$$
\left|x-u_{0}\right|_{0} \leqq \text { (constant) }\left|y-u_{0}\right|_{0}, \quad \epsilon \in\left(0, \epsilon_{0}\right], \quad \mu \in\left[0, \mu_{0}\right],
$$

will now be derived.

First observe that by (2) and (6), if $M$ is a bound for $b(v, w)$ then

$$
\left|(\epsilon \mathfrak{Q}+\mu C+I)^{-1} v\right|_{0} \leqq(M / \delta)|v|_{0}, \quad v \in V_{0} .
$$

Also, by the definitions of $a$ and $S$,

$$
\left|a S^{-1} v\right|_{0} \leqq|v|_{0}, \quad v \in V_{0},
$$

and it follows from (7) that (since $\left.\left|v_{\gamma}=\right| S^{\gamma_{v}}\right|_{0}$ )

$$
\left|C S^{-\gamma_{v}}\right|_{0} \leqq k|v|_{0}, \quad v \in V_{0} \text {. }
$$

Now, (9) implies that

$$
x=u_{0}-(\epsilon Q+\mu C)(\epsilon Q+\mu C+I)^{-1} u_{0}
$$

and by (10)

$$
y=u_{0}-\left(\epsilon S+\mu S^{\gamma}\right)\left(\epsilon S+\mu S^{\gamma}+I\right)^{-1} u_{0}
$$

so

$$
x-u_{0}=(\epsilon Q+\mu C)(\epsilon Q+\mu C+I)^{-1}\left(\epsilon S+\mu S^{\gamma}+I\right)\left(\epsilon S+\mu S^{\gamma}\right)^{-1}\left(y-u_{0}\right) .
$$

Thus, using (11)-(13),

$$
\begin{aligned}
\left|\mathbf{|} x-u_{0}\right|_{0} \leqq & \left|(\epsilon Q+\mu C)(\epsilon Q+\mu C+I)^{-1}\left(y-u_{0}\right)\right|_{0} \\
& +\left|(\epsilon Q+\mu C)(\epsilon Q+\mu C+I)^{-1}\left(\omega S+\mu S^{\gamma}\right)^{-1}\left(y-u_{0}\right)\right|_{0} \\
= & \left|y-u_{0}-(\epsilon Q+\mu C+I)^{-1}\left(y-u_{0}\right)\right|_{0} \\
& +\left|(\epsilon Q+\mu C+I)^{-1}(\epsilon Q+\mu C)\left(\omega S+\mu S^{\gamma}\right)^{-1}\left(y-u_{0}\right)\right|_{0} \\
\leqq & {[1+(M / \delta)]\left|y-u_{0}\right|_{0}+(\epsilon M / \delta)\left|a S^{-1}\left(\epsilon I+\mu S^{\gamma-1}\right)^{-1}\left(y-u_{0}\right)\right|_{0} } \\
& +(\mu M / \delta)\left|C S^{-\gamma}\left(\omega S^{1-\gamma}+\mu I\right)^{-1}\left(y-u_{0}\right)\right|_{0} \\
\leqq & {[1+(M / \delta)]\left|y-u_{0}\right|_{0}+(\epsilon M / \delta)\left|\left(\epsilon I+\mu S^{\gamma-1}\right)^{-1}\left(y-u_{0}\right)\right|_{0} } \\
& +(\mu k M / \delta)\left|\left(\epsilon S^{1-\gamma}+\mu I\right)^{-1}\left(y-u_{0}\right)\right|_{0} \\
\leqq & {[1+(k+2) M / \delta]\left|y-u_{0}\right|_{0 .} . }
\end{aligned}
$$

It remains to estimate $\left|y-u_{0}\right|$ o. Let $u_{0} \in V_{\tau}$ with $\tau \in[0,1]$ and let $E$ be the resolution of the identity for the selfadjoint operator $S$. Then 


$$
\begin{aligned}
\left|y-u_{0}\right|_{0}^{2}= & \left|\left[\left(\epsilon S+\mu S^{\gamma}+I\right)^{-1}-I\right] u_{0}\right|_{0}^{2} \\
= & \int_{0}^{\infty} \frac{\left(\epsilon \lambda+\mu \lambda^{\gamma}\right)^{2}}{\left(\epsilon \lambda+\mu \lambda^{\gamma}+1\right)^{2}}\left(E(d \lambda) u_{0}, u_{0}\right)_{0} \\
\leqq & 2 \epsilon^{2 \tau} \int_{0}^{\infty} \lambda^{2 \tau} \frac{(\epsilon \lambda)^{2-2 \tau}}{(\epsilon \lambda+1)^{2-2 \tau}}\left(E(d \lambda) u_{0}, u_{0}\right)_{0} \\
& +2 \int_{0}^{\infty} \frac{\mu^{2} \lambda^{2 \gamma}}{\left(\mu \lambda^{\gamma}+1\right)^{2}}\left(E(d \lambda) u_{0}, u_{0}\right)_{0} .
\end{aligned}
$$

Now $u_{0} \in V_{\tau}=D\left(S^{r}\right)$ if and only if $\int_{0}^{\infty} \lambda^{2 r}\left(E(d \lambda) u_{0}, u_{0}\right)_{0}<\infty$. So by Lebesgue's dominated convergence theorem, the first term on the right-hand side is $O\left(\epsilon^{2}\right)$ if $\tau=1$ and $o\left(\epsilon^{2 \tau}\right)$ if $\tau<1$, and if $\tau \geqq \gamma$ the second term is $O\left(\mu^{2}\right)$. If $0 \leqq \tau<\gamma$ the second term on the right-hand side is dominated by

$$
2 \mu^{2 \tau / \gamma} \int_{0}^{\infty} \lambda^{2 \tau} \frac{\left(\mu \lambda^{\gamma}\right)^{(2 \gamma-2 \tau) / \gamma}}{\left(\mu \lambda^{\gamma}+1\right)^{(2 \gamma-2 \tau) / \gamma}}\left(E(d \lambda) u_{0}, u_{0}\right)_{0}
$$

which is $o\left(\mu^{2 \tau / \gamma}\right)$. The theorem follows from (8), (14), and these estimates.

Observe that an explicit estimate for $\left|u-u_{0}\right|_{0}$ in terms of given parameters, $\left|u_{0}\right|_{r}$, and $\left|u_{0}\right|_{\gamma}$ is obtainable from the proof of the theorem.

3. The preceding theorem will now be applied to singular perturbation of elliptic boundary value problems with homogeneous Dirichlet boundary conditions. The terminology of the theory of Bessel potentials will be used (cf. Aronszajn and Smith [2], and Adams, Aronszajn and Smith [1]).

Let $m^{\prime} \geqq l>m$ be nonnegative integers and let $D \subset R^{n}$ be a bounded domain of class $C^{2 m^{\prime}}$. Recall that for such domains and any $\alpha \geqq 0$ the Bessel potential spaces $P^{\alpha}(D)$ and $\check{P}^{\alpha}(D)$ coincide up to equivalent norms (cf. [1]). Denote the closure of $C_{0}^{\infty}(D)$ in $P^{\alpha}(D)$ by $P_{0}^{\alpha}(D)$ and the antidual of $P_{0}^{\alpha}(D)$ by $P^{-\alpha}(D) . P^{-\alpha}(D)$ can be realized as a space of distributions on $D$. For $v, w \in P_{0}^{m^{\prime}}(D)$, let

$$
a(v, w)=\sum_{|i|,|j| \leq m^{\prime}} \int_{D} a_{i j}(x) D_{j} v \overline{D_{i} w} d x
$$

with $a_{i j} \in C^{|i|}(\bar{D})$ and

$$
c(v, w)=\sum_{|i|,|j| \leqslant l} \int_{D} c_{i j}(x) D_{j} v \overline{D_{i} w} d x
$$


with $c_{i j} \in C^{|i|}(\bar{D})$. For $v, w \in P_{0}^{m}(D)$, let

$$
b(v, w)=\sum_{|i|,|j| \leq m} \int_{D} b_{i j}(x) D_{j v} \overline{D_{i} w} d x
$$

with $b_{i j} \in C^{|i|}(\bar{D})$.

Let $f_{e, \mu} \equiv f, f_{0} \in P^{-m}(D)$ and let $|v|_{\alpha, D},(v, w)_{\alpha, D}$ denote the norm and inner product in $P^{\alpha}(D)$ respectively. Let $V=P_{0}^{m^{\prime}}(D), V_{0}=P_{0}^{m}(D)$ and assume that (1) and (2) hold. It follows that there is a unique solution $u \in P_{0}^{m^{\prime}}(D)$ of

$$
\epsilon a(u, v)+\mu c(u, y)+b(u, v)=(f, v)_{-m, D} \equiv L(v), \quad v \in P_{0}^{m^{\prime}}(D),
$$

and a unique solution $u_{0} \in P_{0}^{m}(D)$ of

$$
b\left(u_{0}, v\right)=\left(f_{0}, v\right)_{-m, D} \equiv L_{0}(v), \quad v \in P_{0}^{m}(D) .
$$

According to [5, Proposition 6.1 and Theorem 6.2], $V_{1}=D(Q)$ is $P^{2 m^{\prime}-m}(D) \cap P_{0}^{m}(D)$ with an equivalent norm and, for $0 \leqq \tau<$ $1 / 4\left(m^{\prime}-m\right), V_{\tau}$ is $P^{m+2\left(m^{\prime}-m\right) \tau}(D) \cap P_{0}^{m}(D)$ with an equivalent norm. Similarly, $D(C) \supset P^{2 l-m}(D) \cap P_{0}^{l}(D)$ and $C$ maps $P^{2 l-m}(D) \cap P_{0}^{l}(D)$ into $P_{0}^{m}(D)$ continuously. Thus (6) is satisfied and so is (7) with $\gamma=(l-m) /\left(m^{\prime}-m\right)$. Moreover, for any $\theta \in[0,1 / 2)$, if $f_{0} \in P^{-m+\theta}(D)$ then $u_{0} \in P^{m+\theta}(D) \cap P_{0}^{m}(D)$ (cf. Lions and Magenes [11]). Hence it follows from the above theorem that if $\theta \in[0,1 / 2), f_{\theta} \in P^{-m+\theta}(D)$, and $\left|f-f_{0}\right|_{-m, D}=o\left(\epsilon^{\theta / 2\left(m^{\prime}-m\right)}\right)+o\left(\mu^{\theta / 2(l-m)}\right)$, then

$$
\left|u-u_{0}\right|_{m, D}=o\left(\epsilon^{\theta / 2\left(m^{\prime}-m\right)}\right)+o\left(\mu^{\theta / 2(l-m)}\right) .
$$

The theorem also applies to other differential problems with smooth boundary conditions for which the conditions (6) and (7) are satisfied.

\section{REFERENCES}

1. R. D. Adams, N. Aronszajn and K. T. Smith, Theory of Bessel potentials. II, Ann. Inst. Fourier (Grenoble) 17 (1967), no. 2, 1-135. MR 37 \#4281.

2. N. Aronszajn and K. T. Smith, Theory of Bessel potentials. I, Ann. Inst. Fourier (Grenoble) 11 (1961), 385-475. MR 26 \#1485.

3. N. Aronszajn, Quadratic forms on vector spaces, Proc. Internat. Sympos. Linear Spaces (Jerusalem, 1960), Jerusalem Academic Press, Jerusalem; Pergamon Press, Oxford, 1961, pp. 29-87. MR 25 \#4334.

4. A. Friedman, Singular perturbations for partial differential equations, Arch. Rational Mech. Anal. 29 (1968), 289-303. MR 37 \#1754.

5. W. M. Greenlee, Rate of convergence in singular perturbations, Ann. Inst. Fourier (Grenoble) 18 (1968), no. 2, 135-191. MR 39 \#3133.

6. - A two parameter perturbation estimate, Proc. Amer. Math. Soc. 24 (1970), 67-74. 
7. D. Huet, Phénomènes de perturbation singulière dans les problèmes aux limites, Ann. Inst. Fourier (Grenoble) 10 (1960), 61-150. MR 22 \#9737.

8. - Perturbations singulières et régularitê, C. R. Acad. Sci. Paris, Ser A-B 266 (1968), A924-A926. MR 38 \#1383.

9. - Perturbations singulières et régularite, C. R. Acad. Sci. Paris, Ser. A-B 266 (1968), A1237-A1239. MR 38 \#1384.

10. J. L. Lions, Espaces intermédiares entre espaces hilbertiens et applications, Bull. Math. Soc. Sci. Math. Phys. R. P. Roumaine 2(50) (1958), 419-432. MR 27 $\# 1812$.

11. J. L. Lions and E. Magenes, Problèmes aux limites non homogènes et applications. Vol. 1, Dunod, Paris, 1968.

12. R. E. O'Malley, Jr., Topics in singular perturbations, Advances in Math. 2 (1968), 365-470. MR 38 \#382.

Northwestern University, Evanston, Illinois 60201 